\title{
REMARKS ON THE MORSE INDEX THEOREM ${ }^{1}$
}

\author{
WILLIAM T. REID
}

The present note is occasioned by the recent paper of $\mathrm{H}$. Osborn [12] concerning a proof of the Morse index theorem that, in his words, "eliminates the ad hoc subdivisions of the classical proof." Its purpose is to give a brief historical survey of some earlier proofs of the index theorem, and to point out that alternate approaches to the corrections of [13] are to be found in the literature.

Whereas Morse's development of the notion of (negative) type number, (see [9], [10], [11]), did involve the use of broken extremals and a dichotomy of the interval domain resulting in the study of an associated quadratic form in a finite number of variables, it is to be noted that the index theorem has also been established by the following methods which do not employ this device.

1. Methods based upon the theory of differential equations and associated boundary problems. Such a method, depending basically upon the extremizing properties of the proper values of the associated accessory boundary problem (see, for example, Bliss $[2, \S 91]$ ), was employed by K.-S. Hu [7] in his 1932 Dissertation. Hu treated specifically the accessory differential system for a variational problem of Bolza type, and his proof of the continuity of the proper values as functions of the involved interval required the problem to be normal on every subinterval $[a, c]$ of the initial interval $[a, b]$. For certain types of selfadjoint integro-differential problems this same geveral method has been used by Reid [15], (see also [14, §4]), with a modification of proof and altered definition of focal point that preserved the result of the index theorem without any normality restrictions. The availability of this method for more general systems of ordinary differential equations has also been pointed out recently in Reid [16].

2. Methods involving the concept of "natural isoperimetric condition," (as introduced by G. D. Birkhoff and M. R. Hestenes [1]). In this method the type number appears as the minimum number of such isoperimetric conditions which suffice to reduce the given variational problem to a minimizing problem. For a brief discussion of this method, with some alteration of method of attack and details, the

Received by the editors October 2, 1967 and, in revised form, February 5, 1968.

1 This research was supported by the Air Force Office of Scientific Research, Office of Aerospace Research, United States Air Force, under Grant AF-AFOSR-749-65. 
reader is referred to Hestenes [4]. For specific variational problems, related detailed treatments appear in various Ph.D. Dissertations directed by Hestenes, notably those of K. Hazard [3], W. Karush [8], and L. Ritcey [17]. Of direct relevance is $\$ 10$ of [4], which is devoted to a comprehensive survey of results on indices of the second variation. The results presented therein are a combination of the results obtained by Birkhoff and Hestenes [1] and Hazard [3]; in particular, there is given a proof that the index defined in terms of natural isoperimetric conditions is equivalent to the definition of index as the dimension of a maximal linear subspace on which the second variation functional is positive.

3. Methods wherein the Morse index theorem appears as a special instance of a more general theory of focal points for an appropriate class of quadratic forms in Hilbert space (as developed by Hestenes [5, in particular, $\$ \S 16,17] ;[6])$.

An alternative to the proof of Nirenberg concerning the existence of an $r$-dimensional subspace $H^{-}$on which $I$ is negative definite is essentially contained in [1]. In particular, for Osborn's problem, the Lemma 5.2 of [1] establishes the existence of vector functions $v_{1}, \cdots, v_{r}$ of class $C^{\prime \prime}$ such that $I[u]$ is negative definite on the linear set spanned by these functions, and with the aid of well-known approximation theorems these functions may be replaced by a "nearby set" of class $C^{\infty}$.

It is to be noted that both Birkhoff and Hestenes [1] and Osborn [12] deal specifically with the index theorem in the case of a variational problem involving no auxiliary differential equation restraints, and the comments of [13] on the removal of the deficiencies in Osborn's argument are limited to such problems. For variational problems of Lagrange or Bolza type involving differential equations as restraints, and even for such problems which are identically normal, argument of the sort used in Lemma 4.1 of [1] does not in general lead to the conclusion that the involved functions $a_{k}(x)$ have finite right- and left-hand limits at intermediate focal points. Consequently, in these more general problems there appears to be a distinct advantage for a method depending upon continuity properties of the proper values of an associated boundary problem, as in the specific cases considered by $\mathrm{Hu}$ [7] and Reid [15], or for a method employing a more general functional setting as in Hestenes [5], [6].

\section{REFERENCES}

1. G. D. Birkhoff and M. R. Hestenes, Natural isoperimetric conditions in the calculus of variations, Duke Math. J. 1 (1935), 198-286. 
2. G. A. Bliss, Lectures, on the calculus of variations, Univ. of Chicago Press, Chicago, Ill., 1946.

3. K. Hazard, Index theorems for the problem of Bolza in the calculus of variations, Contributions to the calculus of variations, Univ. of Chicago Press, Chicago, Ill., 1938-1941, pp. 293-356.

4. M. R. Hestenes, The problem of Bolza in the calculus of variations, Bull. Amer. Math. Soc. 47 (1942), 57-75.

5. - Application of the theory of quadratic forms in Hilbert space to the cal culus of variations, Pacific J. Math. 1 (1951), 525-581.

6. - Quadratic variational theory and linear elliptic partial differential equations, Trans. Amer. Math. Soc. 101 (1961), 306-350.

7. K.-S. Hu, The problem of Bolza and its accessory boundary value problem, Contributions to the calculus of variations, Univ. of Chicago Press, Chicago, Ill., 19311932, pp. 361-444.

8. W. Karush, Isoperimetric problems and index theorems in the calculus of variations, Dissertation, Univ. of Chicago, Chicago, Ill., 1942.

9. M. Morse, The foundations of the calculus of variations in the large in m-space (Part I), Trans. Amer. Math. Soc. 31 (1929), 379-404.

10. - A generalization of the Sturm separation and comparison theorems in nspace, Math. Ann. 103 (1930), 52-69.

11. - The calculus of variations in the large, Amer. Math. Soc. Colloq. Publ., Vol. 18, Amer. Math. Soc., Providence, R. I., 1934.

12. H. Osborn, The Morse index theorem, Proc. Amer. Math. Soc. 18 (1967), 759762.

13. Correction to my paper "The Morse index theorem," Proc. Amer. Math. Soc. 20 (1969), 337-338.

14. W. T. Reid, Boundary value problems of the calculus of variations, Bull. Amer. Math. Soc. 43 (1937), 633-666.

15. - An integro-differential boundary value problem, Amer. J. Math. 60 (1938), 257-292.

16. - - Variational methods and boundary problems for ordinary linear differential systems, Proc U. S.-Japan Seminar on Differential and Functional Equations, held at Univ. of Minnesota, June 26-30, 1967, pp. 267-299.

17. L. Ritcey, Index theorems for discontinuous problems in the calculus of variations, Dissertation, Univ. of Chicago, Chicago, Ill., 1945.

\section{UNIVERSITY OF OKLAHOMA}

